Mise au point

\title{
Traitement des effets tardifs après radiothérapie : quoi de neuf ?
}

Treatment of radiation-induced late effects: what's new?

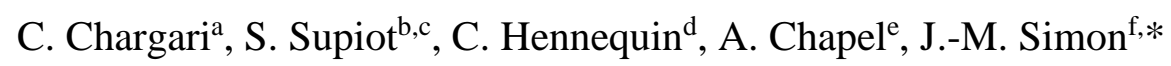

${ }^{a}$ Département de radiothérapie, Gustave-Roussy Cancer Campus, Villejuif, 94800, France

${ }^{\mathrm{b}}$ Département d'oncologie radiothérapie, institut de cancérologie de l'ouest, Boulevard Jacques Monod, 44805 Saint Herblain cedex, France

${ }^{c}$ Université de Nantes, Centre de Recherche en Cancéro-Immunologie de Nantes-Angers, INSERM U1232, CNRS ERL 6001, Institut de Recherche en Santé de l'Université de Nantes 8 quai Moncousu - BP 70721 - 44007 Nantes cedex 1

d Service de Cancérologie-Radiothérapie, Hôpital Saint-Louis, 1, avenue Claude Vellefeaux, 75475 Paris, France

e Institut de Radioprotection et de Sûreté Nucléaire (IRSN), Service de Recherche en Radiobiologie et en Médecine régénérative, Laboratoire de Radiobiologie des expositions Médicales, 31 Avenue de la Division Leclerc, 92260 Fontenay-aux-Roses, France

${ }^{\text {f }}$ Sorbonne Université, Service d'oncologie radiothérapie, AP-HP, Hôpital Pitié-Salpêtrière, F75651 Paris cedex 13, France

Auteur correspondant : JM Simon

Service d'oncologie radiothérapie, Hôpital Pitié-Salpêtrière, F-75651 Paris cedex 13, France

Tel : 0142178161 ; télécopieur : 0142178130

jean-marc.simon@aphp.fr

Titre courant : Effets tardifs après radiothérapie 


\section{RÉSUMÉ}

Les mécanismes des lésions radio-induites tardives sont la résultante de phénomènes multiples et complexes, avec de nombreux acteurs cellulaires et tissulaires intriqués. Le continuum biologique entre effets aigus et effets tardifs après irradiation sera décrit, avec en premier lieu une rupture d'homéostasie qui va conduire à des redistributions cellulaires. De nouveaux éclairages sur la toxicité tardive seront enfin abordés. La radiosensibilité individuelle est un facteur primordial dans le développement des toxicités tardives, et les cliniciens ont un besoin urgent de disposer de tests prédictifs qui permettraient de proposer une radiothérapie réellement personnalisée. Une mise au point sera faite sur les différents tests fonctionnels et génétiques actuellement en cours de validation. La prise en charge des effets secondaires de la radiothérapie reste un problème fréquent de l'oncologue radiothérapeute, et un point sera fait sur les traitements qui peuvent être proposés dans certaines situations cliniques particulières. Enfin, une prise en charge innovante sera développée pour les patients présentant des effets secondaires importants après radiothérapie pelvienne, la greffe de cellules souches mésenchymateuses, avec la présentation du protocole PRISME actuellement ouvert au recrutement des patients.

Mots clés : radiothérapie ; toxicité radio-induite ; radiosensibilité ; effets secondaires de la radiothérapie ; cellules souches mésenchymateuses 


\begin{abstract}
Mechanisms of late radio-induced lesions are the result of multiple and complex phenomena, with many entangled cellular and tissue factors. The biological continuum between acute and late radio-induced effects will be described, with firstly a break in homeostasis which will lead to cellular redistributions. New insights into late toxicity will finally be addressed. Individual radiosensitivity is a primary factor for the development of late toxicities, and clinicians urgently need predictive tests to offer truly personalized radiation therapy. An update will be made on the various functional and genetic tests currently being validated. The management of radio-induced side effects remains a frequent issue for radiation oncologists, and an update will be made for certain specific clinical situations. Finally, an innovative management for patients with significant side effects after pelvic radiotherapy will be developed, involved mesenchymal stem cell transplantation, with the presentation of PRISME protocol currently open to patient's recruitment.
\end{abstract}




\section{Mécanismes des lésions radio-induites tardives}

\subsection{Lésions radio-induites}

Les lésions radio-induites des tissus sains font intervenir des mécanismes multiples et complexes, avec de nombreux acteurs cellulaires et tissulaires intriqués. Les effets observés après irradiation résultent de mécanismes directs (mort mitotique, apoptose), mais également de mécanismes indirects : mécanismes inflammatoires et immunologiques, cascade de coagulation, expression de cytokines proinflammatoires et profibrotiques, remaniements vasculaires, hypoxie [1]. La nature et la sévérité de l'effet clinique dépend cependant de l'organe irradié et de ses spécificités : organisation tissulaire (en parallèle ou en série, tissu compartimental ou non compartimental), degré de différenciation, capacité proliférative et de régénération. La toxicité tardive (ex : fibrose ou hypoplasie) est classiquement la conséquence d'une atteinte des tissus non compartimentaux (ex : système nerveux central, tissus conjonctifs) à renouvellement lent (alpha/beta faible).

A l'échelle cellulaire, les cellules saines peuvent être classées schématiquement selon leur radiosensibilité à la mort mitotique ou apoptotique radio-induite : les cellules végétatives intermitotiques qui se divisent régulièrement et sont peu différenciées (érythroblastes, cellules cryptiques intestinales, spermatogonies, myélocytes) présentent une radiosensibilité élevée et sont principalement impliqués dans la toxicité aiguë de l'irradiation. Les fibroblastes, les cellules différenciées ne se divisant pas régulièrement (cellules hépatiques) ou les cellules différenciées des épithéliums stratifiés présentent une radiosensibilité intermédiaire. Les cellules postmitotiques fixées ne se divisant plus et très différenciées (myocytes, cellules nerveuses, cellules endothéliales) ont une faible radiosensibilité à la mort radio-induite mais peuvent être impliquées dans la toxicité tardive [2]. Cependant la toxicité tardive de la radiothérapie ne doit en effet pas être analysée que comme une destruction directe des cellules 
irradiées mais plutôt comme étant un mécanisme actif résultant d'une perte d'homéostasie tissulaire et entraînant le tissu irradié dans un cycle associant inflammation, perte de fonction, hypoxie, sclérose, faisant interagir des lésions des tissus parenchymateux et des lésions des tissus de soutien [3].

Les facteurs liés à la nature du rayonnement sont également multiples (dose, volume, énergie, étalement, dose par fraction, débit de dose, utilisation d'agents radiosensibilisants) mais ne seront pas étudiés ici. C'est également le cas des facteurs de toxicité liés au patient : troubles métaboliques, microcirculatoires, déficiences des mécanismes de réparation, présence de cofacteurs de risque (tabagisme), statut immunitaire, statut nutritionnel, existence de maladies inflammatoires chroniques [4].

\subsection{Une rupture d'homéostasie}

Il existe un continuum biologique entre effets aigus et effets tardifs. Lors d'une irradiation, le stress oxydatif généré va pouvoir entrâner des modifications directes des cellules épithéliales parenchymateuses, avec altérations de fonction et/ou mort cellulaire. Le modèle de l'irradiation pulmonaire montre bien ces mécanismes, avec en quelques semaines destruction des pneumocytes de type 1 impliqués dans les échanges gazeux au niveau de l'épithélium alvéolaire et prolifération compensatrice des pneumocytes de type II, précurseurs des pneumocytes de type I et impliqués dans la sécrétion du surfactant. L'irradiation entraîne également des modifications endothéliales : apoptose des cellules endothéliales, réduction de la densité microvasculaire et diminution de l'oxygénation tissulaire [5]. L'activation de la voie HIF (hypoxia inducible factor) $-1 \alpha$ et $-2 \alpha$ en réponse à l'afflux de radicaux libres entraîne la sécrétion de VEGF (vascular endothelial growth factor), conduisant à une prolifération endothéliale réactionnelle. L'augmentation de l'expression endothéliale de ICAM1 (intercellular adhesion molecule 1), associée au relargage par les cellules irradiées de nombreux 
signaux moléculaires associés aux lésions radiques (DAMPs : damage associated molecular pattern molecules) dans le microenvironnement, entraîne le recrutement de cellules immunitaires effectrices : macrophages, neutrophiles, lymphocytes. La sécrétion d'ICAM-1 et de sélectines favorise également les agrégats plaquettaires, entrainant des phénomènes ischémiques et une extravasation de liquide extracellulaire augmentant la pression interstitielle du microenvironnement (et donc favorisant l'hypoxie). Les infiltrats leucocytaires au niveau du poumon irradié contribuent au remodelage tissulaire par la sécrétion de cytokines proinflammatoires : interleukine (IL)-1, IL-3, IL-6, IL-7. La sécrétion de TGF- $\beta$ (transforming growth factor beta) et de TNF- $\alpha$ alpha (tumor necrosis factor alpha) entraîne une activation des fibrocytes en fibroblastes prolifératifs. On observe également une activation de la voie du VEGF via la sécrétion du TGF- $\beta$ (par la voie de signalisation SMAD3) [6]. Il se crée ainsi une boucle autocrine impliquant les cellules endothéliales, les macrophages, et les fibroblastes, alors que s'installe un processus inflammatoire et ischémique chronique auto-entretenu, puisque l'hypoxie contribue au stress oxydatif et à l'activation de la voie TGF- $\beta$. Cela entraîne une modification structurelle durable de l'architecture de l'épithélium alvéolaire pulmonaire et de la physiologie des échanges membranaires. Lorsque ces phénomènes perdurent dans le temps, il s'installe une fibrose définitive et parfois progressive, les phénomènes de transition épithélio-mésenchmateuse qui accompagnent ces modifications contribuant au développement d'une fibrose pulmonaire.

Il a été montré une corrélation entre l'intensité de ces modifications aigues et les remaniements tardifs. Ainsi, l'inactivation de la voie ICAM-1 chez les animaux permet de diminuer la réponse inflammatoire aiguë à 5 semaines, et à des temps plus tardifs (18 mois) il est observé une réduction de l'épaisseur des septas alvéolaires irradiés, une diminution du processus de fibrose, et une amélioration de la fonction respiratoire des animaux [7]. Au plan anatomopathologique, la fibrose se caractérise par une perte de l'architecture tissulaire et une accumulation de 
collagène et un remodelage de la matrice extra-cellulaire. Le TGF- $\beta$ (qui joue un rôle oncogène à certains stades tardifs de l'oncogenèse) a un rôle central dans ce mécanisme fibrotique. Lors de l'exposition à un stress radique continu, l'épithélium lésé va sécréter du TGF- $\beta$ qui va interagir avec les fibrocytes quiescents du microenvironnement. L'activation de la méthyltransférase Dbmt1 va entraîner très rapidement (en quelques heures) des modifications épigénétiques au niveau du promoteur du gène de l'inhibiteur de RAS (RASAL-1 inhibitor), et donc l'activation de la voie RAS à l'origine d'une prolifération myofibroblastique soutenue. Cela conduit à une réparation fibrotique avec balance excédentaire de la matrice extra-cellulaire [8]. L'étude des mécanismes de l'entérique radique montre que la structure même de la matrice extracellulaire est modifiée, avec induction de gènes du collagène I, III, IV, VI et VIII, ainsi que de nombreux autres gènes impliqués dans cette balance excédentaire : PA-inhibitor 1 (plasminogen activator inhibitor), TIMP1 et 2, métallo-protéases MMP 1,2,3, SPARC, tenascine-C [9]. Le rôle du stroma dans la toxicité tardive est cependant dual, et il a dans certains cas été montré une fonction radioprotectrice. Ainsi, la thrombomoduline est un facteur anticoagulant exprimé à la surface des cellules endothéliales et stromales dans la moelle osseuse. Son interaction avec la protéine PC (plasma zymogène protéine C) présent dans la matrice extracellulaire contribue à la régénération des cellules souches hématopoïétiques et progénitrices hématopoïétiques après irradiation médullaire [10].

\subsection{Redistributions cellulaires}

Outre les modifications de la matrice extracellulaire, les séquelles de la radiothérapie peuvent s'expliquer également par une déplétion des cellules souches après irradiation pouvant entraîner la survenue d'une perte de fonction tardive. Ainsi, les conséquences physiopathologiques de l'irradiation cérébrale incluent des modifications de la substance blanche (leucoencéphalopathie) et une diminution de la neurogenèse. On observe des lésions 
microvasculaires avec à la phase précoce un œdème cérébral par augmentation de la perméabilité de la barrière hémato-encéphalique (par apoptose endothéliale), des infiltrats lymphocytaires et une inflammation, et à la phase plus tardive survenue de thromboses et de micro-hémorragies. En sus de ces lésions vasculaires, apparaissent des lésions parenchymateuses à type de gliose et démyélinisation [11]. La déplétion apoptotique des cellules souches neurales impliquées situées dans les hippocampes et les régions périventriculaires semble directement impliquée dans le retentissement neurocognitif et les troubles de mémorisation spatiale. L'irradiation cérébrale entraine en effet une rapide diminution de la prolifération cellulaire et de la population des neurones immatures. Les cellules souches survivantes conservent par ailleurs un potentiel diminué de prolifération et de régénération [11]. Des données expérimentales montrent que l'injection in situ de cellules souches permettrait d'améliorer la cognition et que cette amélioration est associée à une augmentation de l'épaisseur de la couche granulaire hippocampique et de l'épaisseur corticale. L'injection systémique pourrait restaurer le flux cérébral d'animaux irradiés, suggérant une action sur la microvascularisation cérébrale, les cellules injectées pouvant se transdifférencier, y compris en oligodendrocytes, astrocytes, et cellules endothéliales. La voie du VEGF semble nécessaire à cette transdifférenciation en cellules endothéliales [12]. Le relargage de cytokines proinflammatoires pourrait contribuer à l'inhibition de la neurogenèse hippocampique via une activation microgliale et une astrogliose réactionnelles. L'irradiation s'accompagne en effet d'une augmentation de l'expression de molécules pro-inflammatoires, telles que le TNF $\alpha$, l'interleukine IB, ICAM-1, et la cycloxygénase COX-2. On observe également une activation de facteurs de transcription tels que NFkB. Le stress oxydatif chronique associé à ce processus inflammatoire et à cette gliose réactionnelle pourrait contribuer à la diminution de la neurogenèse. Enfin, bien que le neurone différencié soit considéré comme radiorésistant, il 
s'associe à ces mécanismes des modifications synaptiques, une perte de plasticité neuronale, et des modifications dans l'expression de gènes neuronaux [13].

Les redistributions cellulaires après radiothérapie sont évolutives dans le temps. Ainsi, des données précliniques, obtenues à partir de modèles d'irradiation pulmonaire, permettent de mieux comprendre les mécanismes de la cascade inflammatoire radio-induite à ses différentes étapes ainsi que des mobilisations des populations cellulaires impliquées [14]. Ces travaux montrent les évolutions des cellules immunitaires présentes dans les espaces alvéolaires et les espaces interstitiels évoluent, entre la phase aigüe et la phase tardive de formation de la fibrose radio-induite et de diminution des surfaces alvéolaires. Ces évolutions concernent les monocytes, les neutrophiles et les macrophages pro-inflammatoires. A la phase tardive, ces modifications aboutissent à une augmentation du nombre des macrophages alvéolaires, associée à la sécrétion de cytokines par les cellules $\mathrm{T}$ auxiliaires de type 1 et 2 , et une augmentation des neutrophiles et monocytes dans les espaces alvéolaires. Dans les espaces interstitiels, la sécrétion de cytokines par les LT auxiliaires de type 2 (exemple : TIMP-1) est associée à une augmentation des macrophages interstitiels et leur interaction avec les fibroblastes contribue à la pathogénie radique pulmonaire tardive. L’implication de cette distribution cellulaire dans la pathogénie post-radique est confirmée par la réduction de la fibrose observée lorsqu'on procède à une déplétion des macrophages interstitiels (ex inhibiteur de CSF1R (colony-stimulating factor receptor-1), alors que l'inhibition des macrophages alvéolaires n'a aucun effet [14].

\subsection{Nouveaux éclairages sur la toxicité tardive : le microbiote}

Des données récentes montrent l'implication du microbiote dans la réponse tumorale aux agents immuno-modulateurs, mais également dans la probabilité de survenue de complications radiques digestives. Il existe à l'état physiologique des interactions étroites entre l'épithélium digestif et la flore bactérienne commensale du tube digestif et des modifications de ces liens 
symbiotiques sont susceptibles d'entraîner des symptômes digestifs. En effet les animaux sans flore digestive présentent des altérations dans les tissus lymphoïdes associés aux muqueuses et des déficits de leur immunité cellulaire, en rapport avec une diminution des interactions entre le système immunitaire et les bactéries. En particulier, il existe des récepteurs TLR (toll like receptor) à la surface des cellules épithéliales et leur interaction avec les microbes non pathogènes est un facteur d'homéostasie, alors que la fixation de ligands pathogènes entraînera une réponse immunitaire. Les mécanismes de l'entérite radique associent : effets cytotoxiques sur l'épithélium digestif, mort cellulaire, ruptures des barrières, phénomènes thrombotiques par l'activation de la cascade de coagulation et lésions endothéliales, lésions ulcérées, effets inflammatoires directs. Les interactions entre l'épithélium et la flore digestive sont perturbées par des translocations bactériennes qui potentialisent la réaction inflammatoire ainsi que par une destruction directe de la flore intestinale par l'irradiation, modifiant la distribution entre agents pathogènes et agents non pathogènes [15]. L'administration de lipopolysaccharides chez des animaux permettait de diminuer leur radiosensibilité via des voies de signalisation impliquant le récepteur du TNFa, la cyclooxygénase 2 (COX2), la prostaglandine E2 et l'interleukine. D'autres données montrent que des animaux sans germe présentent un turnover épithélial moindre et résistance accrue à une irradiation corporelle totale. Il semble qu'une protéine (angiopoiétine-like protéine 4) soit impliquée dans cette différence. Les évaluations de traitements probiotiques ou prébiotiques (aliments non digérables activant la flore microbienne) sont également encourageantes en termes de lésions épithéliales radiques [15].

\section{Radiosensibilité individuelle}

\subsection{Variabilité de la radiosensibilité individuelle}

Tous les patients ne développent pas de toxicité et parmi ceux qui le font, la sévérité de la toxicité varie considérablement d'un patient à l'autre [16-18]. La variation individuelle de la 
réponse tissulaire normale pour une dose donnée a été décrite officiellement pour la première fois en 1936 [19], avec la publication de la courbe dose-réponse sigmoïde, associée à une distribution de fréquence presque gaussienne de la sensibilité individuelle pour le développement de télangiectasies cutanées [20]. Par exemple, une étude portant sur la toxicité cutanée radio-induite chez les patientes atteintes de cancer du sein a démontré que la variation interindividuelle de toxicités cutanées radio-induites était relativement importante pour le même traitement [21]. Cela a conduit à l'hypothèse qu'au moins une partie de cette variabilité s'explique par des variantes génétiques communes. Une base génétique pour la variation individuelle est soutenue par l'existence de plusieurs syndromes génétiques rares associés à une hypersensibilité aux radiations. Cependant, les réponses variables observées chez les patients non affectés par l'un de ces rares syndromes, qui sont traités avec des protocoles impliquant des caractéristiques dosimétriques similaires, suggèrent que des facteurs génétiques sous-jacents sont également importants. Pour détecter ces variations de sensibilité individuelles, les travaux se sont orientés dans un premier temps sur des tests fonctionnels non spécifiques avant de se concentrer sur des analyses génomiques portant sur des aspects spécifiques de la réponse tissulaire à l'irradiation.

\subsection{Test fonctionnels}

Le concept de tests fonctionnels prédictifs est discuté depuis de nombreuses années [22]. Ces tests posent le postulat que le type cellulaire dont on va étudier la radiosensibilité reflète la radiosensibilité intrinsèque de l'organe dont on craint la toxicité tardive. Les cellules souches des tissus à l'origine de la toxicité (intestinale, pulmonaire, neuronale...) étant souvent difficiles d'accès, des travaux ont porté sur des cellules plus accessibles comme les fibroblastes ou les lymphocytes du sang circulant. Il faut garder à l'esprit qu'il est possible que la radiosensibilité spécifique d'un tissu donné ne se reflète pas dans les fibroblastes ou les 
lymphocytes en raison de la grande variabilité intrinsèque de chaque tissu.

Les premiers travaux ont porté sur des tests de survie cellulaire comme base pour les tests prédictifs. Plusieurs petites études ont été cohérentes pour suggérer un lien entre la sensibilité aux radiations cellulaires in vitro et la réponse tissulaire $[23,24]$, mais cette approche n'a pas réussi lorsqu'elle a été entreprise à plus grande échelle [25], probablement en raison de la variabilité des tests. Des études pilotes ont montré que la survie clonogénique des fibroblastes après irradiation était associée à la toxicité radio-induite chez les patients [23,26], mais cela n'a pas été confirmé par des études prospectives [25,27]. Les même travaux ont été conduits sur des lymphocytes avec des résultats préliminaires encourageants [28]. Par la suite des tests fonctionnels mesurant l'apoptose des lymphocytes CD8+ après irradiation ont retrouvé une association significative avec le risque de survenue de fibrose mammaire tardive [29] qui a été confirmée dans une étude prospective multicentrique [30].

Après ces travaux se basant sur la mortalité cellulaire après irradiation, d'autres travaux ont porté sur les mécanismes intra-cellulaires mis en route par la radiothérapie en s'attachant particulièrement aux voies de signalisation des dommages de l'ADN. Ainsi, le nombre de foyers $\gamma$-H2AX persistant après radiothérapie a été utilisé pour évaluer la réponse à la $\mathrm{RT}$ dans différentes études rétrospectives [31]. La réparation des cassures de l'ADN engendre la création de micro-noyaux détectables dans les cellules irradiées. Ce test pourrait aussi aider à prédire la toxicité radio-induite tardive [32]. De même, l'évaluation des blocages du cycle cellulaire après irradiation semblent une voie de recherche possible [33,34]. D'autres travaux portant sur les protéines impliquées dans la réparation des cassures double brin de l'ADN après irradiation comme pATM semblent prometteurs [35].

\subsection{Tests génétiques}


Plusieurs études sur les cellules irradiées d'individus apparentés ont étudié l'héritabilité de l'apoptose radio-induite ou des dommages chromosomiques avec des estimations allant de 58 à $82 \%$ [36]. Comme d'autres traits phénotypiques humains, la radiosensibilité est considérée comme un phénotype polygénique complexe déterminé par l'interaction de plusieurs loci. Les recherches actuelles en radiogénomique visent à identifier les variantes génétiques communes associées à la susceptibilité à développer une toxicité. L'hypothèse radiogénomique ne suppose pas que toute la variation phénotypique est due uniquement à la variation génétique de la lignée germinale, mais reconnaît que les changements épigénétiques (hérités et acquis) pourraient également être importants.

Les premières recherches en radiogénomique se sont concentrées sur les études sur les gènes candidats, c'est-à-dire sur l'étude des polymorphismes d'un seul nucléotide (Single Nucleotide Polymorphism, SNP) dans des gènes codant pour des protéines associées à la réponse au rayonnement, comme la réparation de l'ADN et le remodelage tissulaire. Des associations statistiquement significatives ont été signalées pour les SNP dans une variété de gènes candidats [37]. Malheureusement, ces associations n'ont pas été reproduites dans des études de validation [38]. La raison de l'absence de résultats reproductibles pour la plupart des SNP étudiés est en partie due à des lacunes dans la conception et l'exécution de l'étude, telles que l'échec à corriger les valeurs de p pour les tests d'hypothèses multiples. Ces problèmes sont encore aggravés par le biais de publication. Il peut être trop restrictif de rechercher des SNP uniquement dans le nombre relativement petit de gènes qui sont largement acceptés comme étant impliqués dans l'induction et le traitement des dommages par radiothérapie.

En réponse au manque de succès des études sur les gènes candidats SNP, les travaux actuels portent sur l'étude d'association à l'échelle du génome (Genome Wide Association Study, GWAS) qui utilise le fait que les SNP se regroupent en haplotypes. Cela signifie que la majorité des 11 millions de SNP estimés dans le génome humain peuvent être évalués 
indirectement au moyen d'un microréseau qui génotype entre 500000 et quelques millions de «SNP de marquage » bien choisis. Ainsi, le GWAS offre la possibilité de mener une recherche sans hypothèse d'associations à travers le génome entier sans avoir besoin d'une compréhension préalable de la biologie sous-jacente au phénotype d'intérêt. La première étude GWAS a été une petite étude pilote de la dysfonction érectile après radiothérapie du cancer de la prostate [39]. Plutôt que de trouver des associations avec des gènes impliqués dans la reconnaissance et la réparation des dommages à l'ADN, cette étude a identifié un locus dans un gène impliqué dans la fonction normale des tissus, le gène FSHR, qui code pour le récepteur hormonal stimulant les follicules impliqués dans le développement et la fonction des gonades. Cela ne signifie pas que les gènes de réponse aux radiations connus ne jouent pas de rôle dans l'étiologie des toxicités radio-induites, mais suggère que d'autres voies spécifiques aux tissus peuvent également être importantes, soulignant la complexité des processus biologiques étudiés. Une seconde étude GWAS sur des patients atteints de cancer de la prostate a permis d'identifier plusieurs loci en lien avec la toxicité urinaire, la dysfonction érectile et les saignements rectaux [40]. Une méta-analyse récente des données individuelles de six études portant sur 3871 patients traités par radiothérapie pour un cancer de prostate a identifié trois loci: rs17055178 en lien avec un saignement rectal, rs10969913 en lien avec la dysurie et rs11122573 en lien avec l'hématurie [41]. De manière similaire, une étude GWAS a permis de déterminer des loci impliqués dans la susceptibilité à une toxicité cérébrale de l'irradiation [42].

\subsection{Le futur}

Des premiers résultats sont encourageants. Toute la difficulté actuelle consiste à reproduire les résultats déjà obtenus dans des cohortes internationales et à déterminer les modifications de la prise en charge thérapeutique pour les patients présentant un facteur prédictif de radiotoxicité. 
L'amélioration des collaborations entre équipes de recherche, le recrutement d'un nombre toujours plus important de patients dans des cohortes prospectives, les progrès en génétique, bio-informatique et intelligence artificielle devraient aider à obtenir des réponses claires relatives à la pertinence clinique de ces tests. Le Radiogenomics Consortium a été créé pour permettre la collaboration et le partage des données nécessaires pour obtenir les grands échantillons dans le but d'identifier les biomarqueurs génomiques et de développer des tests fonctionnels prédictifs de l'évolution des effets indésirables après la radiothérapie [43] à travers notamment le projet REQUITE [44]. La radiogénomique étudiant le lien possible entre la variation génotypique et la réponse à l'irradiation a donc le double potentiel de réduire la toxicité et de permettre l'escalade de dose, en personnalisant la radiothérapie en fonction de la sensibilité normale des tissus [45]. En cas de succès, les travaux devraient non seulement déboucher sur des stratégies visant à réduire l'impact négatif de ces toxicités sur les patients cancéreux, mais également à alléger la charge financière imposée au système de santé pour soigner les personnes souffrant de lésions radio-induites. À l'avenir, des analyses génétiques prédictives de la radiosensibilité tumorale et de la radiosensibilité tissulaire normale serviraient d'approches complémentaires pour personnaliser le traitement du cancer.

\section{Prise en charge des effets secondaires de la radiothérapie}

Grâce aux nombreux progrès techniques réalisés durant les vingt dernières années, la survenue de complications graves après radiothérapie est devenue un événement rare. Mais leur prise en charge est souvent complexe, et c'est insister ici sur leur meilleur traitement qui est préventif : rigueur dans la qualité des irradiations, respect des contraintes dosimétriques et contrôle régulier en cours de traitement de la reproductibilité de la dosimétrie initiale.

Certaines toxicités simulent les complications habituelles d'un organe. Par exemple, les complications cardiaques de la radiothérapie (coronaropathies, valvulopathies, troubles du 
rythme) doivent être traitées de la même manière que celles observées sans irradiation préalable. Par contre, d'autres sont plus spécifiques à l'irradiation et nécessitent parfois une prise en charge particulière. Ce sont elles que nous allons développer ici.

\subsection{Fibrose et Nécrose}

Quels que soient la dose et le volume traité, un certain degré de fibrose, dite cicatricielle, s'installe souvent après irradiation. Mais celle-ci n'est réellement gênante cliniquement que dans de rares cas.

\subsubsection{La fibrose : un phénomène partiellement dynamique}

Le développement de la fibrose est caractérisé par une évolution lente, sur plusieurs années qui peut déboucher à terme sur des séquelles irréversibles, avec classiquement trois phases [46]:

- une phase inflammatoire, durant plusieurs mois après l'irradiation

- une phase de fibrose pré-atrophique

- $\quad$ une phase de fibrose atrophique, pouvant amener à la destruction du tissu sous-jacent Lors de ces différentes phases, des processus biologiques ont lieu, qu'ils soient vasculaires ou d'origine fibroblastique, qui peuvent être influencés par des traitements spécifiques. La fibrose radio-induite se caractérise en effet par la transformation de fibroblastes en myofibroblastes avec accumulation excessive de collagènes ou d'autres composants de la matrice extracellulaire. Le processus fibrotique fait intervenir de nombreuses molécules, et en particulier le TGF $\alpha$, mais d'autres protéines, comme celles de la famille Rho (GTPases) sont également impliquées [47].

Dans un essai randomisé contre placebo, mais n'incluant que 22 patients, la combinaison pentoxyfilline-vitamine $\mathrm{E}$ a permis une régression significative de la fibrose radio-induite chez des patientes irradiées pour un cancer du sein [48]. 
La modulation des protéines Rho peut être réalisée par les statines. Un essai de phase II incluant 60 patients présentant une fibrose radio-induite après irradiation ORL, a évalué la pravastatine avec pour but de diminuer la fibrose [49]. Dix-huit patients ont stoppé le traitement avant un an. Une régression de la fibrose a été observée dans 35\% des cas. Au total, si des pistes existent permettant d'envisager un traitement de la fibrose radio-induite, aucune preuve formelle de l'intérêt de ces traitements n'existe aujourd'hui.

\subsubsection{Une fibrose particulière : la pneumopathie interstitielle [50]}

Comme pour la fibrose cutanée, il existe trois phases menant à la fibrose pulmonaire : pendant l'irradiation, une atteinte directe des capillaires pulmonaires et des pneumocytes, souvent asymptomatique, puis une phase inflammatoire, survenant dans les 6 mois après l'irradiation (pneumopathie radique), qui va se compliquer progressivement par une pneumopathie interstitielle. La sévérité clinique dépend du volume irradié. A côté de cette pneumopathie post-radique habituelle, survenant dans le champ d'irradiation, peut survenir une pneumopathie organisée, à distance du champ d'irradiation, de mécanisme physiopathologique non éclairci. Elle n'est observée qu'après irradiation de cancers du sein. Il n'existe pas de traitement reconnu de la pneumopathie post-radique : les corticoïdes sont souvent prescrits à la phase précoce, et atténuent les symptômes. Mais on ignore si la corticothérapie prévient la survenue de la fibrose. C'est insister sur la prévention de sa survenue, en respectant absolument les contraintes de dose (le V20 restant le meilleur paramètre de toxicité ultérieure).

\subsubsection{Nécrose cérébrale [51]}

La généralisation des techniques de stéréotaxie pour les tumeurs cérébrales primitives ou secondaires a eu pour conséquence une augmentation de la fréquence des nécroses cérébrales en territoire irradié (plus de 10\% à un an). Nous ne rentrerons pas dans le problème de son diagnostic, parfois difficile à différencier d'une récidive. Les corticoïdes améliorent les 
symptômes via une réduction de l'œdème périlésionnel. Le bevacizumab $(7.5 \mathrm{mg} / \mathrm{kg}$ toutes les trois semaines) permet d'obtenir fréquemment une réponse radiologique, parfois associée à une amélioration des symptômes. L'edaravone (neuro-protecteur anti-oxydant), associée aux corticoïdes permettrait également une amélioration radiologique et clinique. La place de la chirurgie n'a pas fait l'objet d'études contrôlées, de même que l'oxygène hyperbare.

\subsection{Quelques situations cliniques particulières :}

\subsubsection{La rectite radique [52]}

La rectite radique est en fait une mucite particulière. Sa prise en charge n'a pas fait l'objet de recommandations particulières car les études restent limitées, malgré les nombreuses techniques employées. Là encore une phase précoce est décrite (ténesme, diarrhée, troubles de l'exonération), puis une phase chronique, caractérisée essentiellement par des rectorragies et qui peut survenir en l'absence d'une phase aiguée. L'endoscopie est caractéristique (figure 1). L'acide 5-aminosalicylique et les corticoïdes locaux sont largement utilisés, sans preuve formelle de leur efficacité. Les suppositoires de sucrafalte (non disponibles en France) ont été évalués dans des essais contrôlés, mais n'incluant que peu de patients, et diminueraient les symptômes. Le métronidazole (3 x500mg/jour), donné par voie orale, aurait également un intérêt. Les suppositoires d'acide gras à chaîne légère sont efficaces mais uniquement à la phase aiguë.

La coagulation au laser plasma argon est actuellement la première technique endoscopique utilisée dans le traitement de la rectite radique. Elle permet une amélioration des rectorragies dans 80 à $90 \%$ des cas et de la diarrhée et du ténesme dans 60-75\% des cas. Mais il n'est pas dénué d'effets secondaires, parfois graves dans 10\% des cas (ulcérations chroniques voire perforations). Il ne faut donc la proposer qu'en cas de rectorragies sévères, avec un retentissement hématologique. 
En cas d'échecs, des applications de formaline ou oxygénothérapie hyperbare peuvent être proposés avec des résultats parfois spectaculaires.

\subsubsection{Cystite radique [53]}

Là encore, de nombreuses options ont été proposées pour traiter la cystite radique. Par voie orale, le WF10 (immunomodulateur) et le Pentosane polysulfate sodique (Elmiron®) ont montré une certaine efficacité. Le traitement qui apparaît cependant le plus efficace reste l'ablation des télangiectasies par laser-Yag ou mieux par laser plasma argon. Mais les données prospectives manquent et sont moins bien établies que dans la rectite radique.

Les instillations intra-vésicales de formaline sont également efficaces mais peuvent être mal tolérées et doivent être réservées aux formes réfractaires. Par contre, l'oxygène hyperbare donne d'intéressants résultats et devrait être proposé en cas de récidive après deux ou trois séances de laser. Enfin, la cystectomie avec dérivation digestive est à envisager en dernière extrémité.

En cas d'hémorragie massive, après rétablissement d'une hémodynamique stable, il doit être réalisé un lavage vésical massif pour évacuation des caillots suivie d'une irrigation vésicale. Les injections intra-vésicales d'aluminium peuvent alors être proposées, voire des embolisations artérielles.

\subsubsection{Enterite radique [54]}

La pathogénie de l'entérite radique est complexe et associe des lésions de fibrose de la paroi intestinale et des anomalies vasculaires. A la phase aiguée, comportant essentiellement des diarrhées, le loperamide associé à un régime sans résidu amélioré souvent la symptomatologie. Dans le cadre de l'entérite radique chronique, le régime sans résidu est souvent efficace, les carences nutritionnelles, liées à une malabsorption, doivent être compensées. Sur le plan médicamenteux, le loperamide peut être associé au traitement chélateur des sels biliaires tels que la cholestyramine. Des sténoses localisées peuvent faire 
l'objet d'une chirurgie d'exérèse, qui doit être la plus économe possible. Enfin, l'oxygène hyperbare a également donné des résultats intéressants.

\subsection{Conclusion :}

Les traitements des séquelles tardives de la radiothérapie sont limités et dépendent de l'organe atteint. Le niveau de preuve sur ces approches thérapeutiques est faible et il est difficile de donner des recommandations. Leur prévention par une technique d'irradiation rigoureuse doit être mise en avant.

\section{Greffe de cellules souches}

\subsection{Introduction}

Le nombre de personnes atteintes d'un cancer devrait passer de 18,1 millions en 2018 à 26,4 millions en $2030.60 \%$ de ces patients recevront une radiothérapie avec $50 \%$ de chance de guérison (données du Centre international de recherche sur le cancer, http://gco.iarc.fr). L'irradiation des tissus sains en radiothérapie demeure un problème majeur car elle peut entraîner des effets secondaires très variables d'une personne à l'autre en fonction de la sensibilité individuelle, de l'étendue de la région à traiter et de la dose totale d'irradiation délivrée. Dans la majorité des cas, ces effets se produisent pendant le traitement et les quelques semaines qui suivent ; mais chez une minorité de patients, des effets secondaires dits "tardifs", peuvent apparaître plusieurs mois à plusieurs années après traitement. Jusqu'à dix pour cent d'entre eux développeront des complications gastro-intestinales graves tardives (maladie radio-induite pelvienne - MRIP). Les symptômes sont des proctopathies (5 à $20 \%)$ et des cystites radio-induites $(3,5 \%)$ qui affectent la qualité de vie. Les conséquences sont un coût croissant pour la société (hospitalisations répétées pour soins palliatifs) et un problème éthique pour aider ces patients avec une qualité de vie dégradée de façon irréversible [55]. 
Les symptômes les plus courants pour les rectites radiques sont la diarrhée, le ténesme, la perte de mucus et de sang par le rectum, l'incontinence et les douleurs. Une détérioration progressive est induite par une réduction de la densité des petits vaisseaux sanguins (vascularisation réduite) et une inflammation chronique des tissus. Le tissu normal est progressivement remplacé par un tissu fibreux dense (fibrose), jusqu'à ce que l'oxygénation soit insuffisante pour permettre aux tissus de conserver une fonction normale. Ces dommages progressifs et tardifs induits par les radiations peuvent atteindre un point critique où les tissus se décomposent pour former un ulcère ou un tissu nécrotique. Les symptômes les plus courants pour la cystite radique est une inflammation hémorragique de la muqueuse des voies urinaires. La cystite radique a tendance à être cliniquement grave et peut provoquer une douleur extrême, une hématurie et des symptômes de miction irritants. Les effets tardifs des radiations ionisantes peuvent se manifester dans les couches profondes du muscle de la vessie et provoquer une aggravation progressive de l'endartérite (inflammation de l'intima d'une ou de plusieurs artères) [52].

Les complications chroniques associées à la radiothérapie pelvienne, sont causées par une inflammation persistante qui détruit progressivement le rectum et la vessie et finit par provoquer les affections suivantes : fibrose, ulcères, durcissement des tissus, sténose, occlusion, fistules, perforations et septicémie [55]. La pathologie est similaire à celle des maladies inflammatoires de l'intestin comme la maladie de Crohn.

\subsection{Thérapie cellulaire des séquelles chroniques des radiothérapies: un nouveau traitement thérapeutique des complications réfractaires aux traitements actuels}

Les cellules stromales mésenchymateuses (CSM) auraient la capacité de contrôler l'inflammation par la sécrétion de facteurs anti-inflammatoires et leur activité pro- 
angiogénique protègerait la micro-vascularisation. Grâce à ces deux processus, la thérapie par

CSM limite la formation de fibroses tissulaires, d'ulcères et de sténoses [56].

\subsubsection{Rationnel biologique}

Les études précliniques dans des modèles animaux de troubles gastro-intestinaux graves radio-induits (similaires à la pathologie humaine) ont démontré que lorsque les CSM sont injectées par voie intraveineuse ; elles prolongent la vie des animaux, réduisent la proctite radio-induite (amélioration des selles et diminution des saignements), réduisent l'ulcération par l'amélioration de la musculature (moins de zone nécrotique) et de la vascularisation (moins d'hémorragie). Elles augmentent la capacité de réépithélialisation des muqueuses et diminuent l'hypersensibilité viscérale mécanique (douleur). Elles contrôlent l'inflammation systémique et locale et réduisent l'étendue de la fibrose. Les CSM ont une présence transitoire, ne persistant pas dans l'organisme hôte. Il a été démontré qu'elles limitent la progression tumorale et qu'elles sont sans toxicité [57].

Le traitement des affections post-radiques par la thérapie cellulaire pourrait donc apporter un bénéfice thérapeutique lorsque les autres traitements ont échoué.

Au niveau clinique, l'efficacité thérapeutique des CSM est testée dans le traitement de pathologies qui présentent des symptômes digestifs similaires à ceux observés dans la MRIP. Les CSM sont utilisés dans des essais de phase 1 à 3 pour les maladies inflammatoires chroniques de l'intestin (MICI) et les troubles viscéraux associés à la maladie du greffon contre l'hôte (GVHD) (https://clinicaltrials.gov/).

\subsubsection{Cellules souches et radiothérapie}

Au cours des dix dernières années, les CSM ont été administrées à des patients ayant subi une radiothérapie pour des indications très diverses, notamment l'aplasie de la moelle osseuse, le syndrome hémorragique grave et les complications digestives causées par la radiothérapie. Plus de 8 patients souffrant de brulures radiologiques graves ont été traités avec succès grâce 
à des injections de CSM [58]. Ce traitement a été utilisé avec succès comme thérapie alternative pour les complications chroniques liées à la radiothérapie du cancer du sein chez les femmes pour lesquelles les méthodes conventionnelles avaient échoué [59]. Cette approche a permis d'éliminer la douleur et de guérir complètement les complications chez 57 patientes [60].

\subsubsection{Données de l'essai clinique de phase I d'Epinal}

Dans un essai clinique de phase I, nous avons traité quatre patients par injection de CSM, alors qu'ils avaient reçu une dose excessive de radiation (accident d'Epinal) [61] lors d'une radiothérapie conformationnelle pour un adénocarcinome de la prostate [62]. Entre 2005 et 2006, 24 patients atteints d'un cancer de la prostate ont reçu des doses totales comprises entre 81 Gy et $120 \mathrm{~Gy}$, pour doses prescrites de $69 \mathrm{~Gy}$ à $78 \mathrm{~Gy}$, correspondant à une surexposition de $13 \%$ à $54 \%$. Douze patients ont été grièvement blessés. Ils ont développé une nécrose pelvienne et une fistule vésico-rectale, nécessitant une colostomie et une dérivation urinaire permanente. Sept patients ont eu des complications rectales CTCAE v3 grade 3 ou 4 , et 4 patients ont une proctite et une cystite de grade 1. Dix patients sont décédés de nécrose pelvienne. Quatre patients souffrant d'hémorragie, de douleur rectale, et de signes de rectite radique sévère, réfractaire aux traitements symptomatiques ont été inclus dans cette étude de phase I et traités par l'injection par voie intraveineuse de CSM allogéniques. La douleur, l'hémorragie, la fréquence des diarrhées et des fistules ainsi que les sous-ensembles de lymphocytes dans le sang périphérique ont été évalués avant le traitement par CSM et pendant le suivi. Deux patients ont eu une amélioration de leur symptomatologie douloureuse et une diminution de la fréquence des hémorragies après l'administration de CSM. Chez un patient, la douleur est réapparue après 6 mois et a de nouveau répondu de manière substantielle lors d'une seconde perfusion de CSM. La fréquence des diarrhées douloureuses a diminué de 6/j à 
3/j après la première et à $2 / \mathrm{j}$ après la deuxième injection de CSM chez un patient. Un processus de fistulisation a pu être arrêté chez un patient, ce qui a permis une rémission stable pendant plus de trois ans. Chez tous les patients, le cancer de la prostate est resté en rémission complète et stable. Il a été observé une modulation des sous-ensembles lymphocytaires vers un schéma de régulation et une diminution des cellules $\mathrm{T}$ activées lors de la réponse clinique. Le traitement par la CSM a été efficace sur la douleur, la diarrhée, l'hémorragie, l'inflammation, la fibrose et la fistulisation limitée. Aucune toxicité n'a été observée [62]. Pour les patients atteints de maladies inflammatoires chroniques intestinales réfractaires, les injections systémiques de CSM représentent une option de plus en plus étudiée [63], ou pour la cystite hémorragique [64].

Une revue systématique des essais cliniques de thérapie cellulaire a évalué la sécurité des injections de CSM [65]. Trente-six études ont été recensées et analysées, représentant 1012 patients. Cette méta-analyse n'a détecté aucune complication liée aux injections de CSM. Seule une association significative entre l'administration de CSM et une fièvre passagère a été mise en évidence.

L'expérience que nous avons acquise lors de l'essai de phase I d'usage compassionnel impliquant le recours à la thérapie cellulaire pour traiter des patients surirradiés à Epinal nous a amenés à proposer cette thérapie cellulaire dans le cadre d'un essai thérapeutique pour les patients souffrant d'une rectite ou d'une cystite radique de grade 3 ou 4, lorsque les thérapeutiques usuelles n'ont pas permis d'améliorer la symptomatologie. Cet essai est financé par le Programme de Recherche Clinique Hospitalier.

\subsubsection{Essai clinique de phase II PRISME}

Il s'agit d'un essai thérapeutique de phase II évaluant l'efficacité de l'injection de Cellules Stromales Mésenchymateuses (CSMs) sur la symptomatologie de complications chroniques sévères des radiothérapies abdomino-pelviennes (pelvic radiation disease, PRD), après échec 
des thérapeutiques conventionnelles (PHRC-K 13161, Study protocol number: P130935 ; European Clinical Trials Database (EudraCT) 2014-001462-99, NCT 02814864, https://clinicaltrials.gov). La thérapie cellulaire est allogénique, les CSM issues de la moelle osseuse d'un donneur sont amplifiées sur deux passages, sans subir de manipulation génétique. Le traitement consiste en 3 injections intraveineuses de CSM espacées d'une semaine (figure 1).

Nous proposons ce traitement par thérapie cellulaire aux patients pour lesquels les méthodes conventionnelles, notamment les traitements pharmacologiques, et la photocoagulation à l'argon, ont échoué. L’objectif principal est d'évaluer l'efficacité sur les rectorragies et/ou les hématuries d'une thérapie cellulaire consistant en trois injections intraveineuses allogéniques de CSM de moelle osseuse après l'échec de trois traitements conventionnels (corticostéroïdes ou argon plasma, laser YAG, application de formol) chez les patients atteints de MRIP. Les objectifs secondaires sont d'évaluer la sécurité du traitement et son impact sur la consommation de médicaments analgésiques (analgésiques, opiacés), la fréquence des diarrhées, la qualité de vie et l'intensité de la douleur.

Le critère principal de jugement sera le succès du traitement défini par une diminution d'un grade sur l'échelle LENT SOMA, six mois après la première injection de CSM, les patients éligibles devant présenter des rectorragies ou des hématuries de grade supérieur à 2. Les critères secondaires de jugement seront basés sur la modification des symptomatologies urinaires ou digestives au cours des 6 mois suivant la première injection de CSM, la consommation d'antalgiques, la qualité de vie, et l'humeur. Pour être inclus, les patients devront avoir reçu une radiothérapie pelvienne avec des complications tardives digestives ou urinaires supérieures à un grade 2 après échec d'au moins deux thérapeutiques usuelles. Un bilan devra avoir confirmé que le cancer est en rémission, et que leur état général soit bon (ECOG 0-1). Le recueil de CSM se fait sur un donneur intrafamilial d'au plus 50 ans (âge 
limite pour garantir une viabilité maximale des CSM). La méthodologie est celle d'un essai prospectif de phase II selon un plan en deux étapes (minimax de Simon). Cinq patients devront être inclus au cours de la première étape, et s'il y a au moins un succès, la deuxième étape inclura 7 patients supplémentaires. La production de CSM est assurée par la plate-forme ECellFrance qui comprend deux sites : Établissement Français du Sang (Créteil) et Centre de Transfusion Sanguine des Armées (HIA, Clamart), et les prélèvements et les administrations de CSM seront réalisés dans le département d'hématologie clinique de l'Hôpital SaintAntoine, APHP.

\subsection{Perspectives et conclusions}

Les résultats attendus pour le public seront la guérison des maladies chroniques réfractaires, entraînant une diminution des dépenses de santé en réduisant le traitement et l'hospitalisation des patients et une augmentation de leur qualité de vie.

\section{Conclusion}

Les mécanismes des lésions radio-induites tardives deviennent de mieux en mieux connues, et la radiosensibilité individuelle reste un facteur clé dans le développement des toxicités tardives. A l'heure actuelle, les tests prédictifs de la radiosensibilité individuelle des patients ne sont pas validés et ne peuvent pas être utilisés pour modifier un traitement. Lorsque ces tests auront prouvé leur validité, s'ouvrira alors un nouveau champ de recherche pour savoir comment utiliser les résultats de ces tests pour un patient radiosensible : devra-t-on modifier la dose totale de la radiothérapie, la dose par séance, son étalement ? En attendant, et malgré les progrès des techniques de radiothérapie, il y aura encore des patients à prendre en charge avec des effets secondaires tardifs. Le faible niveau de preuve des traitements actuels laisse l'oncologue radiothérapeute et son patient assez démuni en dehors de traitements 
symptomatiques. La recherche clinique nous apportera peut-être des solutions, avec la greffe de CSM, ou l'étude puis les manipulations du microbiote intestinal, oropharyngé ou cutané, pour lesquels les essais sont en cours.

\section{Déclaration de liens d'intérêts}

Les auteurs déclarent ne pas avoir de liens d'intérêts. 


\section{Références}

[1] De Ruysscher D, Niedermann G, Burnet NG, Siva S, Lee AWM, Hegi-Johnson F. Radiotherapy toxicity. Nat Rev Dis Primers 2019;5:13. https://doi.org/10.1038/s41572-0190064-5.

[2] Rubin P, Casarett GW. Clinical radiation pathology as applied to curative radiotherapy. Cancer 1968;22:767-78. https://doi.org/10.1002/10970142(196810)22:4<767::aid-cncr2820220412>3.0.co;2-7.

[3] Stone HB, Coleman CN, Anscher MS, McBride WH. Effects of radiation on normal tissue: consequences and mechanisms. Lancet Oncol 2003;4:529-36. https://doi.org/10.1016/s1470-2045(03)01191-4.

[4] Chargari C, Kirov KM, Bollet MA, Magné N, Védrine L, Cremades S, et al. Cardiac toxicity in breast cancer patients: from a fractional point of view to a global assessment. Cancer Treat Rev 2011;37:321-30. https://doi.org/10.1016/j.ctrv.2010.08.007.

[5] Vallard A, Rancoule C, Le Floch H, Guy J-B, Espenel S, Le Péchoux C, et al. [Medical prevention and treatment of radiation-induced pulmonary complications]. Cancer Radiother 2017;21:411-23. https://doi.org/10.1016/j.canrad.2017.03.004.

[6] Schaue D, Kachikwu EL, McBride WH. Cytokines in radiobiological responses: a review. Radiat Res 2012;178:505-23. https://doi.org/10.1667/RR3031.1.

[7] Hallahan DE, Geng L, Shyr Y. Effects of intercellular adhesion molecule 1 (ICAM-1) null mutation on radiation-induced pulmonary fibrosis and respiratory insufficiency in mice. $\mathrm{J}$ Natl Cancer Inst 2002;94:733-41. https://doi.org/10.1093/jnci/94.10.733.

[8] Wynn TA, Ramalingam TR. Mechanisms of fibrosis: therapeutic translation for fibrotic disease. Nat Med 2012;18:1028-40. https://doi.org/10.1038/nm.2807.

[9] Strup-Perrot C, Vozenin-Brotons M-C, Vandamme M, Linard C, Mathé D. Expression of matrix metalloproteinases and tissue inhibitor metalloproteinases increases in X-irradiated rat ileum despite the disappearance of CD8a T cells. World J Gastroenterol 2005;11:6312-21. https://doi.org/10.3748/wjg.v11.i40.6312.

[10] Chute JP. To survive radiation injury, remember your aPCs. Nat Med 2012;18:10134. https://doi.org/10.1038/nm.2859.

[11] Greene-Schloesser D, Robbins ME, Peiffer AM, Shaw EG, Wheeler KT, Chan MD. Radiation-induced brain injury: A review. Front Oncol 2012;2:73.

https://doi.org/10.3389/fonc.2012.00073.

[12] Joo KM, Jin J, Kang BG, Lee SJ, Kim KH, Yang H, et al. Trans-differentiation of neural stem cells: a therapeutic mechanism against the radiation induced brain damage. PLoS ONE 2012;7:e25936. https://doi.org/10.1371/journal.pone.0025936.

[13] Balentova S, Adamkov M. Molecular, Cellular and Functional Effects of RadiationInduced Brain Injury: A Review. Int J Mol Sci 2015;16:27796-815. https://doi.org/10.3390/ijms161126068.

[14] Meziani L, Mondini M, Petit B, Boissonnas A, Thomas de Montpreville V, Mercier O, et al. CSF1R inhibition prevents radiation pulmonary fibrosis by depletion of interstitial macrophages. Eur Respir J 2018;51. https://doi.org/10.1183/13993003.02120-2017.

[15] Ferreira MR, Muls A, Dearnaley DP, Andreyev HJN. Microbiota and radiationinduced bowel toxicity: lessons from inflammatory bowel disease for the radiation oncologist. 
Lancet Oncol 2014;15:e139-147. https://doi.org/10.1016/S1470-2045(13)70504-7.

[16] Vale C, Nightingale A, Spera N, Whelan A, Hanley B, Tierney JF. Late complications from chemoradiotherapy for cervical cancer: reflections from cervical cancer survivors 10 years after the national cancer institute alert. Clin Oncol (R Coll Radiol) 2010;22:588-9. https://doi.org/10.1016/j.clon.2010.05.017.

[17] Resnick MJ, Koyama T, Fan K-H, Albertsen PC, Goodman M, Hamilton AS, et al. Long-term functional outcomes after treatment for localized prostate cancer. N Engl J Med 2013;368:436-45. https://doi.org/10.1056/NEJMoa1209978.

[18] Hopwood P, Haviland JS, Sumo G, Mills J, Bliss JM, Yarnold JR, et al. Comparison of patient-reported breast, arm, and shoulder symptoms and body image after radiotherapy for early breast cancer: 5-year follow-up in the randomised Standardisation of Breast Radiotherapy (START) trials. Lancet Oncol 2010;11:231-40. https://doi.org/10.1016/S14702045(09)70382-1.

[19] Holthusen H. Erfahrungen über die Verträglichkeitsgrenze für Röntgenstrahlen and deren Nutzanwendung zur Verhütung von Schäden. Strahlentherapie 1936:254-69.

[20] Burnet NG, Johansen J, Turesson I, Nyman J, Peacock JH. Describing patients' normal tissue reactions: concerning the possibility of individualising radiotherapy dose prescriptions based on potential predictive assays of normal tissue radiosensitivity. Steering Committee of the BioMed2 European Union Concerted Action Programme on the Development of Predictive Tests of Normal Tissue Response to Radiation Therapy. Int J Cancer 1998;79:606-13. https://doi.org/10.1002/(sici)1097-0215(19981218)79:6<606::aidijc9>3.0.co;2-y.

[21] Edvardsen H, Landmark-Høyvik H, Reinertsen KV, Zhao X, Grenaker-Alnæs GI, Nebdal D, et al. SNP in TXNRD2 associated with radiation-induced fibrosis: a study of genetic variation in reactive oxygen species metabolism and signaling. Int $\mathrm{J}$ Radiat Oncol Biol Phys 2013;86:791-9. https://doi.org/10.1016/j.ijrobp.2013.02.025.

[22] Tucker SL, Geara FB, Peters LJ, Brock WA. How much could the radiotherapy dose be altered for individual patients based on a predictive assay of normal-tissue radiosensitivity? Radiother Oncol 1996;38:103-13. https://doi.org/10.1016/0167-8140(95)01669-4.

[23] Burnet NG, Nyman J, Turesson I, Wurm R, Yarnold JR, Peacock JH. Prediction of normal-tissue tolerance to radiotherapy from in-vitro cellular radiation sensitivity. Lancet 1992;339:1570-1. https://doi.org/10.1016/0140-6736(92)91833-t.

[24] Johansen J, Bentzen SM, Overgaard J, Overgaard M. Relationship between the in vitro radiosensitivity of skin fibroblasts and the expression of subcutaneous fibrosis, telangiectasia, and skin erythema after radiotherapy. Radiother Oncol 1996;40:101-9.

https://doi.org/10.1016/0167-8140(96)01777-x.

[25] Peacock J, Ashton A, Bliss J, Bush C, Eady J, Jackson C, et al. Cellular radiosensitivity and complication risk after curative radiotherapy. Radiother Oncol 2000;55:173-8. https://doi.org/10.1016/s0167-8140(00)00173-0.

[26] Geara FB, Peters LJ, Ang KK, Wike JL, Brock WA. Prospective comparison of in vitro normal cell radiosensitivity and normal tissue reactions in radiotherapy patients. Int $\mathbf{J}$ Radiat Oncol Biol Phys 1993;27:1173-9. https://doi.org/10.1016/0360-3016(93)90540-c.

[27] Somaiah N, Chua MLK, Bourne S, Daley F, A’ Hern R, Nuta O, et al. Correlation between DNA damage responses of skin to a test dose of radiation and late adverse effects of earlier breast radiotherapy. Radiotherapy and Oncology 2016;119:244-9. 
https://doi.org/10.1016/j.radonc.2016.04.012.

[28] West CML, Davidson SE, Elyan SAG, Valentine H, Roberts SA, Swindell R, et al. Lymphocyte radiosensitivity is a significant prognostic factor for morbidity in carcinoma of the cervix. International Journal of Radiation Oncology*Biology*Physics 2001;51:10-5. https://doi.org/10.1016/S0360-3016(01)01575-9.

[29] Ozsahin M, Ozsahin H, Shi Y, Larsson B, Würgler FE, Crompton NE. Rapid assay of intrinsic radiosensitivity based on apoptosis in human CD4 and CD8 T-lymphocytes. Int J Radiat Oncol Biol Phys 1997;38:429-40. https://doi.org/10.1016/s0360-3016(97)00038-2.

[30] Azria D, Rosenstein BS, Ozsahin M. Radiation-Induced Side Effects With or Without Systemic Therapies: Prime Time for Prediction of Individual Radiosensitivity. International Journal of Radiation Oncology*Biology*Physics 2008;71:1293-4. https://doi.org/10.1016/j.ijrobp.2008.03.057.

[31] Olive PL, Banáth JP, Keyes M. Residual gammaH2AX after irradiation of human lymphocytes and monocytes in vitro and its relation to late effects after prostate brachytherapy. Radiother Oncol 2008;86:336-46.

https://doi.org/10.1016/j.radonc.2007.09.002.

[32] Barber JB, Burrill W, Spreadborough AR, Levine E, Warren C, Kiltie AE, et al. Relationship between in vitro chromosomal radiosensitivity of peripheral blood lymphocytes and the expression of normal tissue damage following radiotherapy for breast cancer. Radiother Oncol 2000;55:179-86. https://doi.org/10.1016/s0167-8140(99)00158-9.

[33] Hoeller U, Borgmann K, Bonacker M, Kuhlmey A, Bajrovic A, Jung H, et al. Individual radiosensitivity measured with lymphocytes may be used to predict the risk of fibrosis after radiotherapy for breast cancer. Radiotherapy and Oncology 2003;69:137-44. https://doi.org/10.1016/j.radonc.2003.10.001.

[34] Terzoudi GI, Hatzi VI, Barszczewska K, Manola KN, Stavropoulou C, Angelakis P, et al. G2-checkpoint abrogation in irradiated lymphocytes: A new cytogenetic approach to assess individual radiosensitivity and predisposition to cancer. Int J Oncol 2009;35:1223-30. https://doi.org/10.3892/ijo_00000439.

[35] COPERNIC project investigators, Granzotto A, Benadjaoud MA, Vogin G, Devic C, Ferlazzo ML, et al. Influence of Nucleoshuttling of the ATM Protein in the Healthy Tissues Response to Radiation Therapy: Toward a Molecular Classification of Human Radiosensitivity. Int J Radiat Oncol Biol Phys 2016;94:450-60. https://doi.org/10.1016/j.ijrobp.2015.11.013.

[36] West CM, Barnett GC. Genetics and genomics of radiotherapy toxicity: towards prediction. Genome Med 2011;3:52. https://doi.org/10.1186/gm268.

[37] Rosenstein BS. Identification of SNPs associated with susceptibility for development of adverse reactions to radiotherapy. Pharmacogenomics 2011;12:267-75. https://doi.org/10.2217/pgs.10.186.

[38] Barnett GC, Coles CE, Elliott RM, Baynes C, Luccarini C, Conroy D, et al. Independent validation of genes and polymorphisms reported to be associated with radiation toxicity: a prospective analysis study. The Lancet Oncology 2012;13:65-77.

[39] Kerns SL, Ostrer H, Stock R, Li W, Moore J, Pearlman A, et al. Genome-wide association study to identify single nucleotide polymorphisms (SNPs) associated with the development of erectile dysfunction in African-American men after radiotherapy for prostate cancer. Int J Radiat Oncol Biol Phys 2010;78:1292-300. 
https://doi.org/10.1016/j.ijrobp.2010.07.036.

[40] Kerns SL, Stone NN, Stock RG, Rath L, Ostrer H, Rosenstein BS. A 2-stage genomewide association study to identify single nucleotide polymorphisms associated with development of urinary symptoms after radiotherapy for prostate cancer. J Urol 2013;190:102-8. https://doi.org/10.1016/j.juro.2013.01.096.

[41] Kerns SL, Fachal L, Dorling L, Barnett GC, Baran A, Peterson DR, et al. Radiogenomics Consortium Genome-Wide Association Study Meta-Analysis of Late Toxicity After Prostate Cancer Radiotherapy. J Natl Cancer Inst 2020;112:179-90. https://doi.org/10.1093/jnci/djz075.

[42] Wang T-M, Shen G-P, Chen M-Y, Zhang J-B, Sun Y, He J, et al. Genome-Wide Association Study of Susceptibility Loci for Radiation-Induced Brain Injury. JNCI: Journal of the National Cancer Institute 2019;111:620-8. https://doi.org/10.1093/jnci/djy150.

[43] Rosenstein BS. Radiogenomics: Identification of Genomic Predictors for Radiation Toxicity. Semin Radiat Oncol 2017;27:300-9. https://doi.org/10.1016/j.semradonc.2017.04.005.

[44] West C, Azria D, Chang-Claude J, Davidson S, Lambin P, Rosenstein B, et al. The REQUITE project: validating predictive models and biomarkers of radiotherapy toxicity to reduce side-effects and improve quality of life in cancer survivors. Clin Oncol (R Coll Radiol) 2014;26:739-42. https://doi.org/10.1016/j.clon.2014.09.008.

[45] Barnett GC, West CML, Dunning AM, Elliott RM, Coles CE, Pharoah PDP, et al. Normal tissue reactions to radiotherapy: towards tailoring treatment dose by genotype. Nature Reviews Cancer 2009;9:134-42. https://doi.org/10.1038/nrc2587.

[46] Delanian S, Lefaix J-L. The radiation-induced fibroatrophic process: therapeutic perspective via the antioxidant pathway. Radiother Oncol 2004;73:119-31. https://doi.org/10.1016/j.radonc.2004.08.021.

[47] Yarnold J, Brotons M-CV. Pathogenetic mechanisms in radiation fibrosis. Radiother Oncol 2010;97:149-61. https://doi.org/10.1016/j.radonc.2010.09.002.

[48] Delanian S, Porcher R, Balla-Mekias S, Lefaix J-L. Randomized, placebo-controlled trial of combined pentoxifylline and tocopherol for regression of superficial radiation-induced fibrosis. J Clin Oncol 2003;21:2545-50. https://doi.org/10.1200/JCO.2003.06.064.

[49] Bourgier C, Auperin A, Rivera S, Boisselier P, Petit B, Lang P, et al. Pravastatin Reverses Established Radiation-Induced Cutaneous and Subcutaneous Fibrosis in Patients With Head and Neck Cancer: Results of the Biology-Driven Phase 2 Clinical Trial Pravacur. Int J Radiat Oncol Biol Phys 2019;104:365-73. https://doi.org/10.1016/j.ijrobp.2019.02.024.

[50] Hanania AN, Mainwaring W, Ghebre YT, Hanania NA, Ludwig M. RadiationInduced Lung Injury: Assessment and Management. Chest 2019;156:150-62. https://doi.org/10.1016/j.chest.2019.03.033.

[51] Chung C, Bryant A, Brown PD. Interventions for the treatment of brain radionecrosis after radiotherapy or radiosurgery. Cochrane Database Syst Rev 2018;7:CD011492. https://doi.org/10.1002/14651858.CD011492.pub2.

[52] Paquette IM, Vogel JD, Abbas MA, Feingold DL, Steele SR, Surgeons O behalf of the CPGC of TAS of C and R. The American Society of Colon and Rectal Surgeons Clinical Practice Guidelines for the Treatment of Chronic Radiation Proctitis. Diseases of the Colon \& Rectum 2018;61:1135-1140. https://doi.org/10.1097/DCR.0000000000001209. 
[53] Pascoe C, Duncan C, Lamb BW, Davis NF, Lynch TH, Murphy DG, et al. Current management of radiation cystitis: a review and practical guide to clinical management. BJU Int 2019;123:585-94. https://doi.org/10.1111/bju.14516.

[54] Rehailia-Blanchard A, He MY, Rancoule C, Vallard A, Espenel S, Nivet A, et al. [Physiopathology and pharmacological perspectives in the treatment of radiation enteritis]. Cancer Radiother 2019;23:240-7. https://doi.org/10.1016/j.canrad.2018.05.010.

[55] Andreyev HJN, Wotherspoon A, Denham JW, Hauer-Jensen M. "Pelvic radiation disease": new understanding and new solutions for a new disease in the era of cancer survivorship. Scand J Gastroenterol 2011;46:389-97. https://doi.org/10.3109/00365521.2010.545832.

[56] Linard C, Busson E, Holler V, Strup-Perrot C, Lacave-Lapalun J-V, Lhomme B, et al. Repeated autologous bone marrow-derived mesenchymal stem cell injections improve radiation-induced proctitis in pigs. Stem Cells Transl Med 2013;2:916-27. https://doi.org/10.5966/sctm.2013-0030.

[57] François S, Usunier B, Forgue-Lafitte M-E, L'Homme B, Benderitter M, Douay L, et al. Mesenchymal Stem Cell Administration Attenuates Colon Cancer Progression by Modulating the Immune Component within the Colorectal Tumor Microenvironment. Stem Cells Transl Med 2019;8:285-300. https://doi.org/10.1002/sctm.18-0117.

[58] Lataillade JJ, Doucet C, Bey E, Carsin H, Huet C, Clairand I, et al. New approach to radiation burn treatment by dosimetry-guided surgery combined with autologous mesenchymal stem cell therapy. Regen Med 2007;2:785-94. https://doi.org/10.2217/17460751.2.5.785.

[59] Rigotti G, Marchi A, Galiè M, Baroni G, Benati D, Krampera M, et al. Clinical treatment of radiotherapy tissue damage by lipoaspirate transplant: a healing process mediated by adipose-derived adult stem cells. Plast Reconstr Surg 2007;119:1409-22; discussion 14231424. https://doi.org/10.1097/01.prs.0000256047.47909.71.

[60] Maione L, Vinci V, Caviggioli F, Klinger F, Banzatti B, Catania B, et al. Autologous fat graft in postmastectomy pain syndrome following breast conservative surgery and radiotherapy. Aesthetic Plast Surg 2014;38:528-32. https://doi.org/10.1007/s00266-0140311-9.

[61] Peiffert D, Simon J-M, Eschwege F. [Epinal radiotherapy accident: passed, present, future]. Cancer Radiother 2007;11:309-12. https://doi.org/10.1016/j.canrad.2007.09.004.

[62] Voswinkel J, Francois S, Simon J-M, Benderitter M, Gorin N-C, Mohty M, et al. Use of mesenchymal stem cells (MSC) in chronic inflammatory fistulizing and fibrotic diseases: a comprehensive review. Clin Rev Allergy Immunol 2013;45:180-92.

https://doi.org/10.1007/s12016-012-8347-6.

[63] Irhimeh MR, Cooney J. Management of Inflammatory Bowel Disease Using Stem Cell Therapy. Curr Stem Cell Res Ther 2016;11:72-7. https://doi.org/10.2174/1574888x10666150728121738.

[64] Ringdén O, Uzunel M, Sundberg B, Lönnies L, Nava S, Gustafsson J, et al. Tissue repair using allogeneic mesenchymal stem cells for hemorrhagic cystitis, pneumomediastinum and perforated colon. Leukemia 2007;21:2271-6. https://doi.org/10.1038/sj.leu.2404833.

[65] Lalu MM, McIntyre L, Pugliese C, Fergusson D, Winston BW, Marshall JC, et al. Safety of cell therapy with mesenchymal stromal cells (SafeCell): a systematic review and meta-analysis of clinical trials. PLoS ONE 2012;7:e47559. 
https://doi.org/10.1371/journal.pone.0047559.

Figure 1 : Essai clinique de phase 2 évaluant l'efficacité des injections systémiques de CSM pour le traitement des complications abdominales et pelviennes graves et chroniques réfractaires au traitement standard, induites par la radiothérapie (PRISME).

Les patients incluent doivent avoir un grade supérieur à 2 pour la cystite ou la rectite l'inclusion. Ils recevront 3 injections intraveineuses (hebdomadaires) de CSM de 0,8 à 2 millions par $\mathrm{kg}$ et ils seront suivis sur une période de 12 mois. 
Figure 1

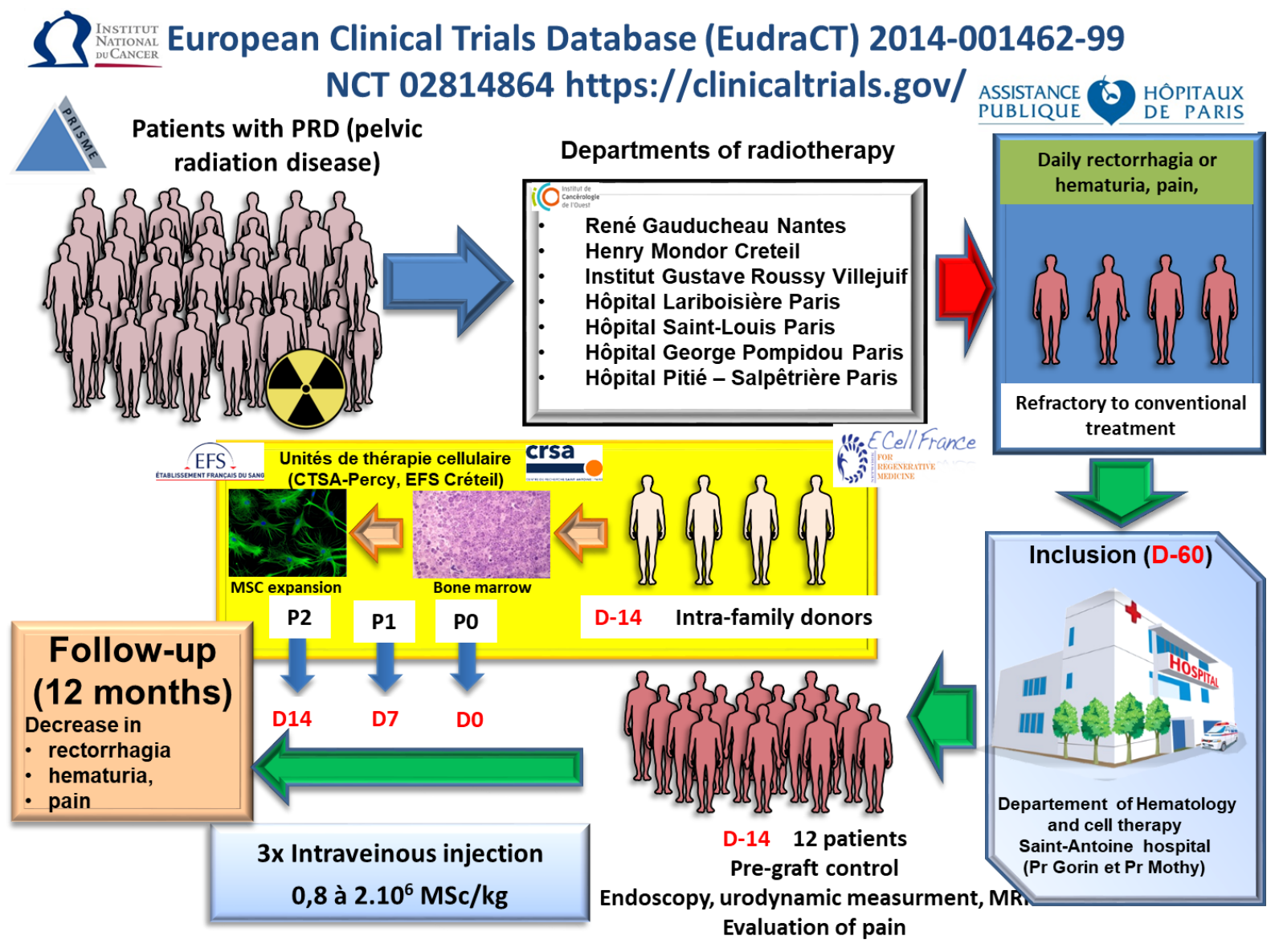

